\title{
Synthesis, Characterization, Effect of Lattice Strain on the Debye-Waller Factor and Debye Temperature of Aluminium Nanoparticles
}

\author{
Endla Purushotham \\ Department of Physics, S R Engineering College, Telangana, India
}

Email address:

psm45456@gmail.com

\section{To cite this article:}

Endla Purushotham. Synthesis, Characterization, Effect of Lattice Strain on the Debye-Waller Factor and Debye Temperature of Aluminium Nanoparticles. American Journal of Nanosciences. Vol. 5, No. 3, 2019, pp. 23-26. doi: 10.11648/j.ajn.20190503.11

Received: October 17, 2019; Accepted: November 9, 2019; Published: November 17, 2019

\begin{abstract}
The synthesis of aluminium (Al) nanocrystalline powder by high-energy ball milling has been investigated. Al powders were ball milled in an argon inert atmosphere. The milled powders were characterized by X-ray diffraction and scanning electron microscopy measurements. The high-energy ball milling of Al after 12 hours resulted in crystallite size (particle size) of about $76 \mathrm{~nm}$. Particle size and lattice strain in Al powder produced by milling have been analyzed by X-ray powder diffraction. The lattice strain $(\varepsilon)$ and Debye-Waller factor $(B)$ are determined from the half-widths and integrated intensities of the Bragg reflections. In this $\mathrm{Al}$, the Debye-Waller factor is found to increase with the lattice strain. From the correlation between the strain and effective Debye-Waller factor, the Debye-Waller factors for zero strain have been estimated for Al. The variation of energy of vacancy formation as a function of lattice strain has been studied.
\end{abstract}

Keywords: X-ray Diffraction, Lattice Strain, Crystallite Size, Debye-Waller Factor, Vacancy Formation Energy

\section{Introduction}

Influence of Tool Revolving on Mechanical Properties of some metals and alloy had been developed as next generation engineering production $[1,2]$ and with some thermal and mechanical properties and engineering applications $[3,4]$. Physical and chemical properties of these materials are highly size dependent. Therefore, it is important to develop novel techniques for the synthesis of nanomaterials. The Debye-Waller factor is an important lattice dynamical property. There is considerable X-ray work on the DebyeWaller factors of Aluminium But it is interesting to study the effect of lattice strains on the Debye-Waller factors of these metals. Inagaki et al [5,6] showed that in several nonmetallic powders, the strains produced during grinding have a significant effect on the Debye-Waller factors measured from X-ray diffraction intensities. Sirdeshmukh et al [7] observed the effect of lattice strains on the Debye-Waller factors in semiconductor powder materials. Gopi Krishna and Sirdeshmukh [8] studied the effect of lattice strains on the Debye-Waller factor of ytterbium metal. In the present investigation the results of a systematic study of the effect of lattice strains on the Debye-Waller factors and crystallite size of face centred cubic $\mathrm{Al}$ metal is reported. These results are being reported for the first time.

\section{Experimental}

A higly pure aluminum obtained from the solid State Physics Laboratory, New Delhi was used. The powder samples were obtained by gently filing highly pure Al metal ingots with a jeweller's file. A part of this powder was used to prepare the initial sample. The remaining powder was subjected to milling in a ball mill for 4, 8, 12, 16 and 20 hours to produce strains and decrease the particle size. X-ray diffractograms were recorded with the initial sample and with samples prepared after each spell of milling. The diffractograms were obtained with a Philips CWU 3710 Xray powder diffractometer in the $2 \theta$ range $20-120^{\circ}$ using filtered $\mathrm{CuK}$. $\square$ at a goniometer speed of $0.5^{\circ}$ per minute and a chart speed of $20 \mathrm{~mm} / \mathrm{min}$. All measurements were made at room temperature. The observed integrated intensities have been corrected for thermal diffuse scattering using the method of Chipman and Paskin [9]. 


\section{Analysis of Data}

For the relative intensity method, the expression for the observed intensities $\mathrm{I}_{0}$ is given by

$$
\mathrm{I}_{0}=\mathrm{CL}_{\mathrm{p}} \mathrm{JF}_{\mathrm{T}}^{2}
$$

where $\mathrm{L}_{\mathrm{p}}$ is the Lorentz-polarization factor, $\mathrm{J}$, the multiplicity factor, $\mathrm{F}_{\mathrm{T}}$ the structure factor and $\mathrm{C}$ is a constant. For a flat powder specimen, the absorption correction is independent of the angle $\theta$, Klug and Alexander [10] and, hence, is lumped with the constant. The structure factor $F_{T}$ in terms of the structure factor $\mathrm{F}$ for the static lattice is given by

$$
\mathrm{F}_{\mathrm{T}}=\mathrm{Fe}^{-\mathrm{M}}
$$

We may also write Eq. (1) as

$$
\mathrm{I}_{0}=I_{c} e^{-2 B\left(\frac{\sin \theta}{\lambda}\right)^{2}}
$$

where $I_{c}$ is the intensity corresponding to the static lattice and is given by

$$
\mathrm{I}_{\mathrm{c}}=\mathrm{L}_{\mathrm{p}} \mathrm{JF}^{2}
$$

For crystals with fec structure, the structure factor $F$ is given by

$$
\mathrm{F}=4 \mathrm{f}
$$

F being the atomic scattering factor. Values of the atomic scattering factor were taken from Cromer and Waber [11] and International Tables for X-ray Crystallography [12], and have been corrected for dispersion, Cromer and Liberman [13]. The choice of the atomic scattering factors was guided by Cromer's [11] recommendation who suggested that the atomic scattering factors given in the International Tables for X-ray Crystallography [12] which are computed from the Hartree-Fock wave functions are the best for the lighter atoms or ions in the periodic table up to $\mathrm{Rb}^{+1}$. For the heavier atoms or ions Cromer recommends the use of the Dirac-slater relativistic scattering factors given by Cromer and Waber [11].

From Eq. (3) it can be seen that $\log \left(\mathrm{I}_{0} / \mathrm{I}_{\mathrm{c}}\right)$ is linearly related to $(\sin \theta / \lambda)^{2}$. By a least square treatment of data, $\mathrm{B}$ was determined. From the Debye-Waller theory

$$
\mathrm{B}=\left(\frac{8 \pi^{2}}{3}\right)<\mathrm{u}^{2}>
$$

for a cubic crystal, where $\left\langle\mathrm{u}^{2}>\right.$ is the mean-square amplitude of vibration. Further, B, may also be expressed as

$$
\mathrm{B}=\left(\frac{6 h^{2}}{m k_{B} T}\right) \mathrm{W}(\mathrm{x})
$$

where $\mathrm{m}$ is the mass, $\mathrm{T}$ the absolute temperature and $\mathrm{h}$ and $\mathrm{k}_{\mathrm{B}}$ are the Planck and the Boltzmann constants respectively. The function $\mathrm{W}(\mathrm{x})$ is given by

$$
\mathrm{W}(\mathrm{x})=\left[\frac{\varphi(x)}{x^{2}}+\frac{1}{4 x}\right]
$$

where $\varnothing(x)$ is the Debye function and $x=\theta_{M} / T, \theta_{M}$ being the Debye temperature. Benson and Gill [14] have tabulated values of $\mathrm{W}(\mathrm{x})$ for a wide range of $\mathrm{x}$ for small increments, from which $\theta_{M}$ can be obtained from the value of $B$.

The total peak broadening $B_{r}$ may be expressed as,

$$
\mathrm{B}_{\mathrm{r}} \cos =\frac{\mathrm{k} \lambda}{\mathrm{t}}+\varepsilon \sin \theta
$$

The plot of $B_{r} \cos \theta / \lambda$ versus $\sin \theta / \lambda$ is a straight line with slope equal to $\varepsilon \square$ and hence the particle size ' $\mathrm{t}$ ' can be estimated from the intercept. Typical Hall-Williamson plot between $B_{r} \cos \theta / \lambda$ and $\sin \theta / \lambda$ is shown in Figure 1 .

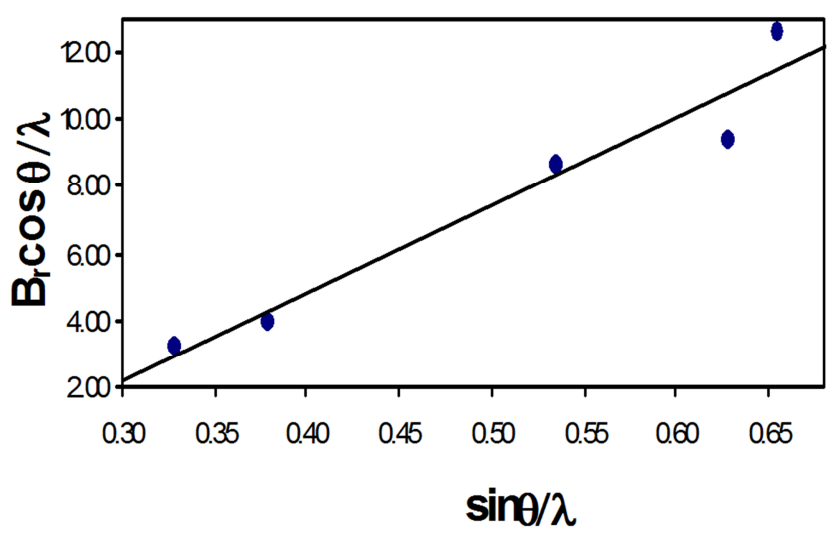

Figure 1. Plot of $B_{r} \cos \theta / \lambda V s \sin \theta / \lambda$ for Al after milling for 12 .

The lattice strains were determined from the plot of $B_{r} c o s$ $\theta / \lambda$ against $\sin \theta / \lambda$ following standard procedures [15]. The measured half-widths were corrected for instrumental broadening with reference to a pure strain-free silicon powder. The variation of particle size with milling time is within the limits of experimental errors. This shows that while the milling is enough to create strains, it affect the particle size to a measurable extent. A typical Hall-Williamson plot is shown in.

\section{Results and Discussion}

The values of the lattice strain, crystallite size, root mean square amplitude of vibrations, Debye-Waller factor and Debye temperature of $\mathrm{Al}$ powders, ground for different durations, obtained in the present study are given in Table 1. As the objective of the present work is to investigate the strain dependence of Debye-Waller factors, the variation of the lattice strain $(\varepsilon)$ and Debye-Waller factor (B) for different milling times for the fcc metal Al is shown in Figure 2. Particle size decrease with milling time. Both lattice strain and DebyeWaller factor increase with milling time. This is similar to the observations of Inagaki et al [5, 6], Sirdeshmuch et al [7] and Gopi Krishna and Sirdeshmukh [8]. The Debye-Waller factor increases with milling time and lattice strain in a slightly nonlinear fashion. Crystallite size decreases with milling time. An 
extrapolation of the $\mathrm{B}$ versus $\varepsilon$ curve to $\varepsilon=0$ gives the values of Debye-Waller factor $0.84 \AA^{2}$ for Al. Zero-strain value of Debye-Waller factor is less than the value for the initial sample. The zero strain Debye-Waller factor of $0.84 \AA^{2}$ for $\mathrm{Al}$ is close to the value of $0.83 \AA^{2}$ obtained by Chipman [1], using single crystal X-ray diffraction. Thus, the Debye-Waller factors of $\mathrm{Al}$ powder samples carry an effect due to lattice strain. While comparing the Debye-Waller factors calculated from the lattice dynamical models with experimental results, Vetelino et al [16] have attributed the difference to inaccuracies in the experimental values caused by neglecting the TDS corrections. The repeated milling of the powder sample leads to lattice

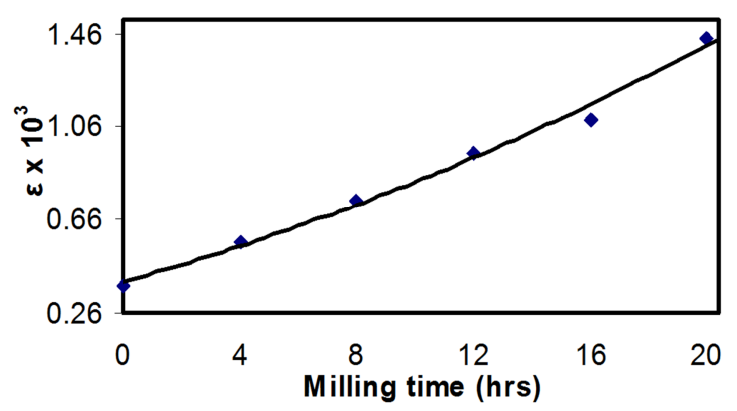

distortion which gives rise to microstrains in the lattice. These microstrains increase the contribution of static component of Debye-Waller factor. Thus both lattice strain and the observed Debye-Waller factor, which is the sum of static and thermal components, increase with milling time. Thus, whenever Debye-Waller factors are determined from X-ray intensities on powder samples, it is desirable to make an estimate of the lattice strain and if the strain is large, a suitable correction is to be made as done in the present study. The Debye temperatures derived from the zero strain values of Debye-Waller factor of $\mathrm{Al}$ is $396 \mathrm{~K}$. The value agree well with the value of $390 \mathrm{~K}[1,2]$ obtained for $\mathrm{Al}$ from the single crystal X-ray diffraction.
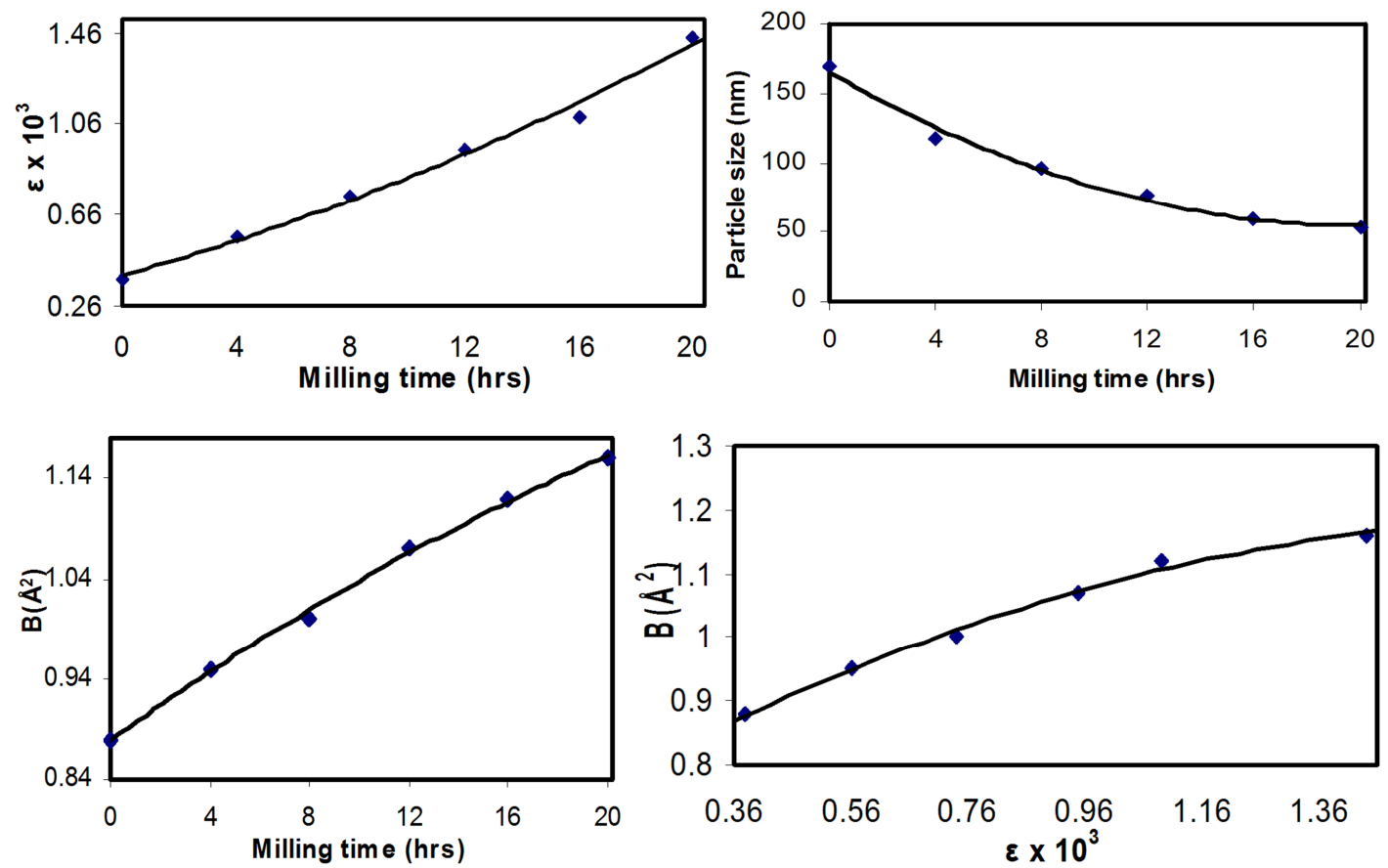

Figure 2. Milling time Vs lattice strain, Crystallite size and Debye-Waller factor and lattice strain Vs Debye-Waller factor curves for Al.

Glyde [17] derived the following relation between the energy of vacancy formation $\left(E_{f}\right)$ and the Debye temperature $(\theta)$ of a solid. The relation is

$$
\mathrm{E}_{\mathrm{f}}=\mathrm{A}(\mathrm{k} / \mathrm{h})^{2} \mathrm{M} \theta^{2} \mathrm{a}^{2}
$$

where $\mathrm{a}$ is the interatomic spacing, A a constant shown to be equal to $1.17 \times 10^{-2}, \mathrm{M}$ the molecular weight and $h$ and $k$ are the Plank's and the Boltzmann's constants, respectively.
Glyde recommended the use of X-ray based values in eq. (5). The validity of eq. (5) was verified for a number of fcc, bcc and hcp metals [18]. Therefore, the X-ray Debye temperatures obtained in the present work have been used to study the variation of vacancy formation energy as a function of lattice strain in Al. The values of vacancy formation energies are also included in Table 1.

Table 1. Values of lattice strain $(\varepsilon)$, particle size $(t)$, Debye-Waller factor $(B)$, root mean square amplitudes of vibration $\left\langle u>\right.$, Debye temperature $\left(\theta_{M}\right)$ and energy of vacancy formation $\left(E_{f}\right)$ of strained Al powders.

\begin{tabular}{|c|c|c|c|c|c|c|c|}
\hline Metal & Grinding time & $\varepsilon \times 10^{3}$ & T (nm) & $<\mathbf{u}>(\AA)$ & B $\left(\AA^{2}\right)$ & $\theta_{M}(K)$ & $E_{f}(e V)$ \\
\hline \multirow{5}{*}{$\mathrm{Al}$} & 0 & 0.38 & 169 & $0.1822(4)$ & $0.88(2)$ & $388(3)$ & 1.41 \\
\hline & 4 & 0.56 & 118 & $0.1913(4)$ & 0.95 (1) & $375(5)$ & 1.30 \\
\hline & 8 & 0.74 & 96 & $0.1932(3)$ & $1.00(1)$ & $363(3)$ & 1.22 \\
\hline & 16 & 1.09 & 59 & $0.2134(4)$ & $1.12(2)$ & $342(9)$ & 1.08 \\
\hline & 20 & 1.44 & 53 & $0.2241(4)$ & $1.16(1)$ & $336(6)$ & 1.05 \\
\hline
\end{tabular}

\section{Conclusion}

Al powders were strained by milling for 20 hours. From a study of X-ray diffractograms recorded at different stages of milling, it is observed that milling for 20 hours has systematic effect on the particle size. However, the milling produces lattice strain and also enhances the effective Debye- 
Waller factor. By an extrapolation of the plot between the Debye-Waller factor and the lattice strain, the zero strain Debye-Waller factors are obtained for Al. The variation of energy of vacancy formation as a function of lattice strain has been studied.

\section{Acknowledgements}

The Authors thank University Grants Commission (UGC), New Delhi, for financial support under Special Assistance Programme no. F. 530/8/DRS/2009 (SAP-I).

\section{References}

[1] V. Ashok Kumar, V. Sammaiah, P. Elsever - Science Direct Materials Today: Proceedings, (2017) 1-7.

[2] P. Srikanth Rao, D. Manoj Kumar, J. Gopikrishna, N. Elsevier ScienceDirect Materials Today, Vol. 5, issue. 1 (1), (2018) 1264-1270.

[3] Ch. Shiva Krishna, Manoj Kumar, J. International Journal of Materials Science, Vol. 12, issue. 4, (2017) 627-633.

[4] P. Satish Kumar, S. R. Sastry, Ch. Devaraju, A. Materials Today Proceedings Elsevier, Vol. 4, issue. 2, (2017) 330-335.

[5] Inagaki, M. Furuhashi, H., T Ozeki et al., J Mater Sci. 6, (1971) 1520.

[6] M Inagaki, H Furuhashi, T Ozeki and S Naka J. Mater. Sci. 8, (1973) 312 .
[7] Sirdeshmukh, D. B., Subhadra, K. G., Hussain, K. A., Gopi Krishna, N., and Rag- havendra Rao. B., Cryst. Res. Technol 28, (1993) 15.

[8] Gopi Krishna, N., and Sirdeshmukh., D. B., Indian J Pure \& Appl Phys. 31, (1993) 198.

[9] Chipman, D. R., and Paskin, A., J. Appl. Phys. 30, (1959) 1938.

[10] Klug, H. P., and Alexander, L. E., (1974). X-ray Diffraction Procedures (John Wiley and Sons, U.S.A.).

[11] Cromer, D. T., and Waber, J. T., Acta Cryst. 18, (1965) 104.

[12] International Tables for X-ray Crystallography (1968) Vol. III (Kynoch Press, Birmingham).

[13] Cromer, D. T., and Liberman, D., J. Chem. Phys. 53, (1970) 1891.

[14] Benson, G. C., and Gill, E. K., (1966) Table of Integral Functions Related to Debye-Waller factor, National Research Council of Canada, Ottawa.

[15] Kaelble, E. F., Handbook of X-rays (New York Mc Graw ill) (1967).

[16] Vetelino, J. F., Gaur, S. P., Mitra, S. S., Phys. Rev. B5, (1972) 2360 .

[17] Glyde, H. R., J. Phys and Chem Solids (G. B) 28, (1967) 2061.

[18] Micro-and Macro-Properties of Solids (Springer Series in Material Science) (2006). 